\title{
Strong chromatic index of products of graphs
}

\author{
Olivier Togni \\ LE2I, UMR CNRS 5158, Université de Bourgogne, BP 47870, 21078 Dijon Cedex, France \\ olivier.togni@u-bourgogne.fr
}

received January 2004, revised November 2006, accepted May 2007.

\begin{abstract}
The strong chromatic index of a graph is the minimum number of colours needed to colour the edges in such a way that each colour class is an induced matching. In this paper, we present bounds for the strong chromatic index of three different products of graphs in terms of the strong chromatic index of each factor. For the Cartesian product of paths, cycles or complete graphs, we derive sharper results. In particular, strong chromatic indices of $d$-dimensional grids and of some toroidal grids are given along with approximate results on the strong chromatic index of generalized hypercubes.
\end{abstract}

Keywords: Strong edge colouring; induced matching; Cartesian product; Kronecker product; strong product.

\section{Introduction}

Let $G=(V, E)$ be a simple graph with vertex set $V$ and edge set $E$. An edge between vertex $x$ and vertex $y$ will be denoted by $x y$.

A proper edge-colouring is a mapping $c: E(G) \rightarrow \mathbb{N}$ satisfying $c(x y) \neq c(y z), \forall x y, y z \in E$. For any vertex $x \in V$, let $S_{c}(x)$ denote the set of the colours of all edges incident to $x$. A proper edge-colouring $c$ is said to be strong if no two edges of the same colour lie on a path of length 3 ; that is, for any edge $x y$ of $G, S_{c}(x) \cap S_{c}(y)=\{c(x y)\}$. Equivalently, a strong colouring also corresponds to a partition of the edges into induced matchings. The strong chromatic index of $G$, denoted by $\chi_{s}^{\prime}(G)$, is the minimum number of colours of any strong colouring of $G$. A strong colouring of a graph $G$ corresponds to a vertex colouring of $L(G)^{2}$, the square of the line-graph of $G$; where the square of a graph is obtained by adding edges between vertices at distance 2 in the graph. A colouring of the square of a graph is also referred to as a distance-2 or $L(1,1)$ colouring in some works.

These problems of strong edge-colouring and distance colouring have interesting applications, specifically for channel assignment in mobile multi-hop radio networks [10] and in cellular networks [4].

The problem of determining the strong chromatic index of a graph is proved to be NP-complete, even for bipartite graphs of girth at least $g$, for any fixed $g$ [6]. In 1985, Erdôs and Nešetřil conjectured (see [2]) that the strong chromatic index of every graph of maximum degree $\Delta$ is at most $\frac{5}{4} \Delta^{2}$. Later, Faudree et al. conjectured in [2] that $\chi_{s}^{\prime}(G) \leq \Delta^{2}$ for bipartite graphs $G$ of maximum degree $\Delta$. In [7], a probabilistic argument is used to show that $\chi_{s}^{\prime}(G) \leq 1.998 \Delta(G)^{2}$. Other approximation results exist, for instance for cubic graphs [1], multigraphs [3] and $C_{4}$-free graphs [5]. Exact values for specific graphs are presented in $[2,8,9]$.

1365-8050 @ 2007 Discrete Mathematics and Theoretical Computer Science (DMTCS), Nancy, France 
In this paper, we study the strong chromatic index of graphs obtained by Cartesian, Kronecker and strong products (definitions are given below). In Section 2, we derive upper bounds for each of them in terms of the strong chromatic index of the two factors along with two lower bounds for the Cartesian product. In Section 3, we turn our attention on the Cartesian product to present improved bounds for products of paths, cycles or cliques. In particular, these results allow to find the exact value of the strong chromatic index for $d$-dimensional grids and some $d$-dimensional toroidal grids and approximate results for other toroidal grids and generalized hypercubes.

The following notation will be used throughout this paper. For a graph $G$, denote by $n_{G}$ its order, by $\Delta(G)$ its maximum degree and by $\chi(G)$ its chromatic number.

The Cartesian product $G \square H$ of two graphs $G$ and $H$ has vertex set $V(G) \times V(H)$ and edge set $\{(a, x)(b, y): a b \in E(G)$ and $x=y$ or $x y \in E(H)$ and $a=b\}$.

The Kronecker (sometimes called direct or categorical) product $G \times H$ has vertex set $V(G) \times V(H)$ and edge set $\{(a, x)(b, y): a b \in E(G)$ and $x y \in E(H)\}$.

The strong product $G \otimes H$ has vertex set $V(G) \times V(H)$ and edge set $E(G \square H) \cup E(G \times H)$.

\section{General bounds}

\subsection{Cartesian product}

Theorem 1 For any graph $G$ and for any graph $H$ that contains two adjacent vertices of maximum degree, we have

$$
\chi_{s}^{\prime}(G \square H) \geq 2 \Delta(G \square H) .
$$

Proof: Let $a b$ be an edge of $G$, with $a$ being a vertex of maximum degree and let $x y$ be an edge of $H$, with both $x$ and $y$ being a vertex of maximum degree. Denote by $S$ the set of all edges incident to $(a, x)$ or $(a, y)$ plus the edge $(b, x)(b, y)$. Then all edges of $S$ must be coloured by distinct colours in any strong colouring. A simple count gives $|S|=2 \Delta(G)+2 \Delta(H)=2 \Delta(G \square H)$.

Theorem 2 Let $G$ and $H$ be two graphs. For the Cartesian product, we have

$$
\chi_{s}^{\prime}(G \square H) \leq \chi_{s}^{\prime}(G) \chi(H)+\chi_{s}^{\prime}(H) \chi(G) .
$$

Proof: Let $G^{\prime}=G \square H$ and let $k_{G}=\chi_{s}^{\prime}(G)$ and $k_{H}=\chi_{s}^{\prime}(H)$. Denote by $c_{G}$ a strong colouring of $G$ with colours from $0,1, \ldots k_{G}-1$ and denote by $c_{H}$ a strong colouring of $H$ with colours from $0,1, \ldots k_{H}-1$. Let $v_{G}$ be a proper vertex colouring of $G$ using the $\chi(G)$ colours $0,1, \ldots \chi(G)-1$ and let $v_{H}$ be a proper vertex colouring of $H$ using the $\chi(H)$ colours $0,1, \ldots \chi(H)-1$.

A colouring $c^{\prime}$ of $G^{\prime}$ is defined as follows:

For any edge $a b$ of $G$, for any vertex $x$ of $H$, set

$$
c^{\prime}((a, x)(b, x))=c_{G}(a b)+k_{G} v_{H}(x),
$$

for any edge $x y$ of $H$, for any vertex $a$ of $G$, set

$$
c^{\prime}((a, x)(a, y))=c_{H}(x y)+k_{H} v_{G}(a)+k_{G} \chi(H) .
$$


As $c_{G}$ and $c_{H}$ are proper colourings and $c^{\prime}((a, x)(b, x))<k_{G} \chi(H)$, then $c^{\prime}$ is a proper colouring too. So it remains to show that $c^{\prime}$ is strong. For any vertex $a$ of $G$ and for any vertex $x$ of $H$, let $I(a, x)=\left\{s+k_{G} v_{H}(x): s \in S_{c_{G}}(a)\right\}$ and let $J(a, x)=\left\{s+k_{H} v_{G}(a)+k_{G} \chi(H): s \in S_{c_{H}}(x)\right\}$.

First, consider two vertices $(a, x)$ and $(b, x)$ of $G^{\prime}$, with $a b \in E(G)$. By the definition of the colouring $c^{\prime}$, we have $S_{c^{\prime}}((a, x))=I(a, x) \cup J(a, x)$, and $S_{c^{\prime}}((b, x))=I(b, x) \cup J(b, x)$. Since $c_{G}$ is strong, we have that $S_{c_{G}}(a) \cap S_{c_{G}}(b)=\left\{c_{G}(a b)\right\}$. Thus $I(a, x) \cap I(b, x)=\left\{c_{G}(a b)+k_{G} v_{H}(x)\right\}$. Since $v_{G}$ is a proper colouring, we have $v_{G}(a) \neq v_{G}(b)$. Thus $J(a, x) \cap J(b, x)=\emptyset$. Therefore $S_{c^{\prime}}((a, x)) \cap$ $S_{c^{\prime}}((b, x))=\left\{c_{G}(a b)+k_{G} v_{H}(x)\right\}=\left\{c^{\prime}((a, x)(b, x))\right\}$.

Next, consider two vertices $(a, x)$ and $(a, y)$ of $G^{\prime}$, with $x y \in E(H)$. By a similar argument, as $v_{H}$ is a proper colouring and $c_{H}$ is a strong colouring, we have $S_{c^{\prime}}((a, x)) \cap S_{c^{\prime}}((a, y))=\left\{c^{\prime}((a, x)(a, y))\right\}$.

Hence we have proved that $c^{\prime}$ is a strong colouring of $G^{\prime}=G \square H$ with $\chi_{s}^{\prime}(G) \chi(H)+\chi_{s}^{\prime}(H) \chi(G)$ colours.

To see how tight the above theorem is, we present the following lower bound in relation with the fractional chromatic number. The fractional chromatic number of a graph $G$ is $\chi_{f}(G)=\min \frac{k}{p}$ for which $G$ has a $p$-tuple $k$-colouring, that is an assignment of $p$ positive integers from a set of $k$ integers (colours) to each vertex of $G$ such that adjacent vertices receive disjoint sets of colours. It is known that $\omega(G) \leq \chi_{f}(G) \leq \chi(G)$ for any graph $G$, where $\omega(G)$ is the clique number of $G$.

Theorem 3 Let $G$ and $H$ be two graphs, then

$$
\chi_{s}^{\prime}(G \square H) \geq \chi_{f}(G) \Delta(H)
$$

Proof: Let $x \in V(H)$ be a vertex of degree $d(x)=\Delta(H)$ and let $S^{x}$ be the subgraph of $H$ consisting of the star of order $\Delta(H)+1$ and center $x$. Remember that the product $G \square H$ contains $n=|V(G)|$ copies $H_{1}, \ldots, H_{n}$ of $H$. Denote by $S_{i}^{x}$ the copy of $S^{x}$ in each copy $H_{i}$ of $H$ and let $S=\bigcup_{i=1}^{m} S_{i}^{x}$.

As each edge of a star $S_{i}^{x}$ must be coloured with a different colour in any strong colouring of $G \square H$, and the colours on two adjacent stars $S_{i}^{x}$ and $S_{i^{\prime}}^{x}$ must be distinct too, one can see that finding a strong edge colouring of $S$ is equivalent to finding a $\Delta(H)$-tuple colouring of $G$. Assume that there exists a $\Delta(H)$-tuple $k$-colouring of $G$. Then,

$$
\chi_{s}^{\prime}(G \square H) \geq \chi_{s}^{\prime}(S) \geq k \geq \chi_{f}(G) \Delta(H),
$$

since by definition, $\chi_{f}(G) \leq \frac{k}{\Delta(H)}$.

In view of this theorem, we can deduce that Theorem 2 gives a upper bound close to the optimal if $\chi_{f}(G)$ is close to $\chi(G)$ and $\chi_{s}^{\prime}(H)$ is close to $\Delta(H)$. For instance, Theorem 2 gives the exact value of the strong chromatic index of the product of a star $S_{n}$ on $n+1$ vertices by a $K_{2}$. For the product $K_{n} \square S_{m}$, with Theorem 2 and Theorem 3, we obtain $n m \leq \chi_{s}^{\prime}\left(K_{n} \square S_{m}\right) \leq n(m+n-1)$. Also, for the Cartesian product of a bipartite graph by itself, if $\chi_{s}^{\prime}(G) \leq \Delta(G)^{2}$ then $\chi_{s}^{\prime}(G \square G) \leq 4 \Delta(G)^{2}=\Delta(G \square G)^{2}$. Thus we obtain infinite families of bipartite graphs verifying the conjecture of Faudree et al..

Nevertheless, Theorem 2 is not optimal for many product graphs. For instance, for the product of two paths $P_{m}$ and $P_{n}$ where $m, n \geq 3$, with Theorem 1 and Theorem 2 we obtain $8 \leq \chi_{s}^{\prime}\left(P_{m} \square P_{n}\right) \leq 12$. In Corollary 4 of Section 3.3 , we will determine the exact value of $\chi_{s}^{\prime}\left(P_{m} \square P_{n}\right)$, showing that the lower bound is tight in general. 


\subsection{Kronecker product}

Theorem 4 Let $G$ and $H$ be two graphs different from $K_{2}$. For the Kronecker product $G \times H$ we have

$$
\chi_{s}^{\prime}(G \times H) \leq \chi_{s}^{\prime}(G) \chi_{s}^{\prime}(H)
$$

Proof: Let $G^{\prime}=G \times H$ and let $k_{G}=\chi_{s}^{\prime}(G)$ and $k_{H}=\chi_{s}^{\prime}(H)$. Denote by $c_{G}$ a strong colouring of $G$ with colours from $0,1, \ldots k_{G}-1$ and denote by $c_{H}$ a strong colouring of $H$ with colours from $0,1, \ldots k_{H}-1$.

A colouring $c^{\prime}$ of $G^{\prime}$ is defined as follows: for any edge $a b$ of $G$, for any edge $x y$ of $H$, set

$$
c^{\prime}((a, x)(b, y))=c_{G}(a b)+k_{G} c_{H}(x y) .
$$

This colouring is clearly proper because $c_{G}$ and $c_{H}$ are both proper and for any edge $e$ of $G, c_{G}(e)<$ $k_{G}$. We have $S_{c^{\prime}}((a, x))=\left\{\alpha+k_{G} \beta: \alpha \in S_{c_{G}}(a), \beta \in S_{c_{H}}(x)\right\}$ and $S_{c^{\prime}}((b, y))=\left\{\alpha+k_{G} \beta: \alpha \in\right.$ $\left.S_{c_{G}}(b), \beta \in S_{c_{H}}(y)\right\}$. Since $c_{G}$ and $c_{H}$ are strong, we have $S_{c_{G}}(a) \cap S_{c_{G}}(b)=\left\{c_{G}(a b)\right\}$ and $S_{c_{H}}(x) \cap$ $S_{c_{H}}(y)=\left\{c_{H}(x y)\right\}$. Hence $S_{c^{\prime}}((a, x)) \cap S_{c^{\prime}}((b, y))=\left\{c_{G}(a b)+k_{G} c_{H}(x y)\right\}=\left\{c^{\prime}((a, x)(b, y))\right\}$. Thus $c^{\prime}$ is a strong colouring of $G \times H$.

This result is optimal for products of stars: If $G=S_{m}$ and $H=S_{n}$, then the product $G \times H$ contains a star $S_{m n}$ and since $\chi_{s}^{\prime}\left(S_{n}\right)=n$, Theorem 4 gives the exact value of $\chi_{s}^{\prime}\left(S_{m} \times S_{n}\right)$. Moreover, for the Kronecker product of a bipartite graph by itself, if $\chi_{s}^{\prime}(G) \leq \Delta(G)^{2}$ then this theorem gives $\chi_{s}^{\prime}(G \times G) \leq \Delta(G)^{4}=\Delta(G \times G)^{2}$. Thus we again obtain infinite families of bipartite graphs verifying the conjecture of Faudree et al.

\subsection{Strong product}

As the edge set of the strong product $G \otimes H$ is the union of the edge set of $G \square H$ and of $G \times H$, we shall construct a strong colouring of $G \otimes H$ by colouring the edges of the Cartesian product $G \square H$ using Theorem 2 and the edges of the Kronecker product $G \times H$ by a modified version of the colouring defined in proof of Theorem 4 . The two next lemmas will ensure us that each of these two colourings remain strong in the final graph $G \otimes H$.

Lemma 1 For any graphs $G$ and $H$, there exist a strong colouring cof $G \square H$ in $\chi_{s}^{\prime}(G) \chi(H)+\chi_{s}^{\prime}(H) \chi(G)$ colours that verifies the following additional property: for any edge ab of $G$ and for any edge $x y$ of $H$, $S_{c}((a, x)) \cap S_{c}((b, y))=\emptyset$.

Proof: The colouring $c$ of $G \square H$ is the same as the colouring $c^{\prime}$ defined in the proof of Theorem 2 Keeping the same notation as for the proof of Theorem 2. let us see that for any edge $a b$ of $G$ and any edge $x y$ of $H$, the equality $S_{c}((a, x)) \cap S_{c}((b, y))=\emptyset$ holds; i-e. that any edge $(a, x)\left(a^{\prime}, x^{\prime}\right)$ incident to vertex $(a, x)$ has a colour different from the colour of any edge $(b, y)\left(b^{\prime}, y^{\prime}\right)$ incident to vertex $(b, y)$. We have four cases to consider:

1. $a a^{\prime} \in E(G)$ and $x=x^{\prime}, b b^{\prime} \in E(H)$ and $y=y^{\prime}$. Then $c^{\prime}\left((a, x)\left(a^{\prime}, x^{\prime}\right)\right)=c_{G}\left(a a^{\prime}\right)+k_{G} v_{H}(x)$ and $c^{\prime}\left((b, y)\left(b^{\prime}, y^{\prime}\right)\right)=c_{G}\left(b b^{\prime}\right)+k_{G} v_{H}(y)$. Since $v_{H}$ is proper and $x y \in E(H), v_{H}(x) \neq v_{H}(y)$ and thus $c^{\prime}\left((a, x)\left(a^{\prime}, x^{\prime}\right)\right) \neq c^{\prime}\left((b, y)\left(b^{\prime}, y^{\prime}\right)\right)$. 
2. $x x^{\prime} \in E(G)$ and $a=a^{\prime}, y y^{\prime} \in E(H)$ and $b=b^{\prime}$. Then $c^{\prime}\left((a, x)\left(a^{\prime}, x^{\prime}\right)\right)=c_{H}\left(x x^{\prime}\right)+k_{H} v_{G}(a)+$ $k_{G} \chi(H)$ and $c^{\prime}\left((b, y)\left(b^{\prime}, y^{\prime}\right)\right)=c_{H}\left(y y^{\prime}\right)+k_{H} v_{G}(b)+k_{G} \chi(H)$. Since $v_{G}$ is proper and $a b \in E(G)$, $v_{G}(a) \neq v_{G}(b)$ and thus $c^{\prime}\left((a, x)\left(a^{\prime}, x^{\prime}\right)\right) \neq c^{\prime}\left((b, y)\left(b^{\prime}, y^{\prime}\right)\right)$.

3. $a a^{\prime} \in E(G)$ and $x=x^{\prime}, y y^{\prime} \in E(H)$ and $b=b^{\prime}$. Then $c^{\prime}\left((a, x)\left(a^{\prime}, x^{\prime}\right)\right)=c_{G}\left(a a^{\prime}\right)+k_{G} v_{H}(x) \neq$ $c_{H}\left(y y^{\prime}\right)+k_{H} v_{G}(b)+k_{G} \chi(H)=c^{\prime}\left((b, y)\left(b^{\prime}, y^{\prime}\right)\right)$.

4. $x x^{\prime} \in E(G)$ and $a=a^{\prime}, b b^{\prime} \in E(H)$ and $y=y^{\prime}$. Then $c^{\prime}\left((a, x)\left(a^{\prime}, x^{\prime}\right)\right)=c_{H}\left(x x^{\prime}\right)+k_{H} v_{G}(a)+$ $k_{G} \chi(H) \neq c_{G}\left(b b^{\prime}\right)+k_{G} v_{H}(y)=c^{\prime}\left((b, y)\left(b^{\prime}, y^{\prime}\right)\right)$.

Therefore, in all cases, $S_{c^{\prime}}((a, x)) \cap S_{c^{\prime}}((b, y))=\emptyset$, which proves the lemma.

Lemma 2 For any graphs $G$ and $H$, there exist a strong colouring $c$ of $G \times H$ in $2 \chi_{s}^{\prime}(G) \chi_{s}^{\prime}(H)$ colours that verifies the following additional property: for any edge ab of $G$ and for any edge $x y$ of $H$,

$$
S_{c}((a, x)) \cap S_{c}((a, y))=\emptyset
$$

and

$$
S_{c}((a, x)) \cap S_{c}((b, x))=\emptyset
$$

Proof: The colouring $c$ of $G \times H$ is obtained by modifying the strong colouring $c^{\prime}$ given in the proof of Theorem 4 in this way: Let $k=\chi_{s}^{\prime}(G) \chi_{s}^{\prime}(H)$ and let $\prec_{G}$ (resp. $\prec_{H}$ ) be any ordering of the vertices of $G$ (resp. of $H$ ). For any edge $a b$ of $G$ with $a \prec_{G} b$ and any edge $x y$ of $H$ with $x \prec_{H} y$, set

$$
c((a, x)(b, y))=c^{\prime}((a, x)(b, y))
$$

and

$$
c((b, x)(a, y))=c^{\prime}((b, x)(a, y))+k .
$$

Keeping the same notation as for the proof of Theorem 4, let us see first that for any edge $a b$ of $G$ and any edge $x y$ of $H$, the equality $S_{c}((a, x)) \cap S_{c}((a, y))=\emptyset$ holds; i-e. that any edge $(a, x)\left(a_{1}, x^{\prime}\right)$ incident to vertex $(a, x)$ has a colour different from the colour of any edge $(a, y)\left(a_{2}, y^{\prime}\right)$ incident to vertex $(b, y)$. Let $m_{1}=c_{G}\left(a a_{1}\right)+k_{G} c_{H}\left(x x^{\prime}\right)$ and let $m_{2}=c_{G}\left(a a_{2}\right)+k_{G} c_{H}\left(y y^{\prime}\right)$. To have $m_{1}=m_{2}$, one needs $c_{G}\left(a a_{1}\right)=c_{G}\left(a a_{2}\right)$ and $c_{H}\left(x x^{\prime}\right)=c_{H}\left(y y^{\prime}\right)$, which is impossible unless $a_{1}=a_{2}$ and $x^{\prime}=y$ and $y^{\prime}=x$. But in that case, we obtain $c^{\prime}\left((a, x)\left(a_{1}, x^{\prime}\right)\right)=m_{1}$ and $c^{\prime}\left((a, y)\left(a_{2}, y^{\prime}\right)\right)=m_{1}+k$ if $a \prec_{G} a_{1}$ and $x \prec_{H} y$, or else $c^{\prime}\left((a, x)\left(a_{1}, x^{\prime}\right)\right)=m_{1}+k$ and $c^{\prime}\left((a, y)\left(a_{2}, y^{\prime}\right)\right)=m_{1}$. Therefore, in all cases, $c^{\prime}\left((a, x)\left(a_{1}, x^{\prime}\right)\right) \neq c^{\prime}\left((a, y)\left(a_{2}, y^{\prime}\right)\right)$. Hence, $S_{c}((a, x)) \cap S_{c}((a, y))=\emptyset$.

Similarly, one can see that $S_{c}((a, x)) \cap S_{c}((b, x))=\emptyset$.

Theorem 5 Let $G$ and $H$ be two graphs. For the strong product $G \otimes H$ we have

$$
\chi_{s}^{\prime}(G \otimes H) \leq \chi_{s}^{\prime}(G) \chi(H)+\chi_{s}^{\prime}(H) \chi(G)+2 \chi_{s}^{\prime}(G) \chi_{s}^{\prime}(H)
$$

Proof: Remember that the edge set of $G \otimes H$ is the union of the edge set of $G \square H$ and of the edge set of $G \times H$. Colour the edges of $G \square H$ with a colouring $c_{1}$ satisfying Lemma 1 using a set $\mathcal{C}$ of $\chi_{s}^{\prime}(G) \chi(H)+$ $\chi_{s}^{\prime}(H) \chi(G)$ colours and colour the edges of $G \times H$ with a colouring $c_{2}$ satisfying Lemma 2 and using a set $\mathcal{C}^{\prime}$ disjoint with $\mathcal{C}$ of $2 \chi_{s}^{\prime}(G) \chi_{s}^{\prime}(H)$ colours. This produces an edge-colouring of $G \otimes H$. Since $\mathcal{C}$ and 
$\mathcal{C}^{\prime}$ are disjoint, this colouring is proper and since $c_{1}$ verifies the additional property of Lemma 1 and $c_{2}$ verifies the additional property of Lemma 2 , this colouring is strong.

Notice that this theorem gives the exact value of the strong chromatic index of the strong product of two complete graphs: since $K_{m} \otimes K_{n}=K_{m n}$ then $\chi_{s}^{\prime}\left(K_{m n}\right)=\left(\begin{array}{c}m n \\ 2\end{array}\right)=\chi_{s}^{\prime}\left(K_{m}\right) \chi\left(K_{n}\right)+\chi_{s}^{\prime}\left(K_{n}\right) \chi\left(K_{m}\right)+$ $2 \chi_{s}^{\prime}\left(K_{m}\right) \chi_{s}^{\prime}\left(K_{n}\right)$.

\section{Cartesian products of paths, cycles and cliques}

In this section, we define the notion of $(k, t)$-colourability that leads us to improved bounds for the product of paths, cycles and complete graphs. Optimal values of the strong chromatic index are found for some graphs.

\section{$3.1(k, t)$-colourable graphs}

Definition 1 Two strong colourings $c_{1}$ and $c_{2}$ of a graph $G$ are compatible if for any vertex $x$ of $G$, $S_{c_{1}}(x) \cap S_{c_{2}}(x)=\emptyset$.

Definition $2 A$ graph $G$ is $(k, t)$-colourable if there exist $t$ strong colourings $c_{i}, 1 \leq i \leq t, c_{i}: E(G) \rightarrow$ $\{1,2, \ldots, k\}$ that are pairwise compatible.
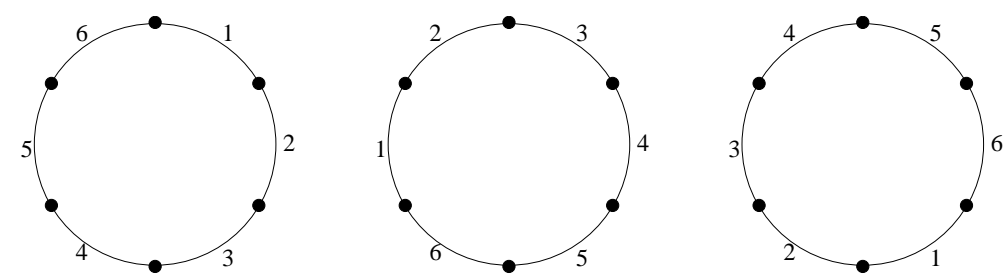

Fig. 1: Three compatible strong colourings of $C_{6}$ in six colours showing that $C_{6}$ is $(6,3)$-colourable.

Notice that a $(k, t)$-colourable graph is also $(\alpha k, \alpha t)$-colourable for any integer $\alpha \geq 1$. In particular, every graph $G$ is $\left(t \chi_{s}^{\prime}(G), t\right)$-colourable for any integer $t \geq 1$.

For the path $P_{n}$ on $n$ vertices, it is known that $\chi_{s}^{\prime}\left(P_{n}\right)=3$ for any $n \geq 4$, thus $P_{n}$ is $(3 t, t)$-colourable. This result can be strengthened as shown in the following proposition.

Proposition 1 For any integers $n \geq 2$ and $t \geq 1$, the path $P_{n}$ is $(4 t, 2 t)$-colourable.

Proof: We only have to show that $P_{n}$ is $(4,2)$-colourable, i-e. that there exist two compatible strong colourings of $P_{n}$ with 4 colours. The first strong colouring $c_{1}$ is defined by giving the colours $1,2,3,4,1$, $2,3,4, \ldots$ to the edges of $P_{n}$, starting from an end-vertex and going to the other end-vertex. The second colouring $c_{2}$ is defined by giving the colours $3,4,1,2,3,4,1,2, \ldots$ to the edges of $P_{n}$ starting from the same end-vertex than for the first colouring.

Proposition 2 For the cycle $C_{n}$, the following holds:

- for any $k \geq 3$ and for any $n \geq 1, C_{k n}$ is $\left(k t,\left\lfloor\frac{k}{2}\right\rfloor t\right)$-colourable, 
- for any $n \geq 5, n \neq 6, C_{n}$ is $(5 t, 2 t)$-colourable.

Proof: First, observe that, given a strong colouring $c$ of $C_{n}$, if for any colour $i$, any two edges of colour $i$ are separated by at least $d-1$ edges along the shortest path (we call such a colouring $d$-distant), then there exist $\left\lfloor\frac{d}{2}\right\rfloor$ compatible strong colourings. These colourings can be obtained from the cycle $C_{n}$ with its colouring $c$ by a rotation of $\frac{4 k \pi}{n}$, for $k=0,1, \ldots,\left\lfloor\frac{d}{2}\right\rfloor-1$.

For the first assertion, a strong $k$-distant colouring is given by assigning cyclically the colours $1,2, \ldots, k$ to the edge of the cycle $C_{k n}$ (see Figure 1 for an example with $k=6$ ).

For the second assertion, observe first that $C_{7}$ and $C_{11}$ are $(5,2)$-colourable, as can be seen by the colourings $1,2,3,4,1,3,5$ and $3,4,1,2,5,4,2$ for $C_{7}$ and $1,2,3,4,1,2,3,4,1,3,5$ and $3,4,1,2,3,4,1$, $2,5,4,2$ for $C_{11}$. Now, for the remaining cases, we have to find a 4-distant colouring using at most 5 colours. Let $n=4 p+i, 0 \leq i \leq 3$. Observe that, as $n>7$ and $n \neq 11$, we have $p \geq i$. The colouring of $C_{n}$ is obtained by repeating $i$ times the pattern $1,2,3,4,5$ then $p-i$ times the pattern $1,2,3,4$ along the cycle.

Proposition 3 For any integers $n \geq 3$ and $t \geq 1$, the complete graph $K_{n}$ is $\left(\frac{n(n-1)}{2} t,\left\lfloor\frac{n}{2}\right\rfloor t\right)$-colourable.

Proof: Consider first the case of even $n$ and let $n=2 p . K_{2 p}$ is decomposable into $2 p-1$ perfect matchings $M_{i}, 0 \leq i \leq 2 p-2$. Order the edges of each matching $M_{i}$ and denote by $e_{i}^{j}$ the $j^{t h}$ edge of matching $M_{i}, 0 \leq j \leq p-1$. Then define the $p$ strong colourings $c_{k}$ of $K_{n}$ as follows: for any edge $e$ of $K_{n}$, for any $k, 0 \leq k \leq p-1$, set

$$
c_{k}\left(e_{i}^{j}\right)=(i+(j+k)(2 p-1)) \bmod p(2 p-1) .
$$

We treat the case of odd $n$ in a similar way. Let $n=2 p+1$. $K_{2 p+1}$ is decomposable into $2 p+1$ matchings $M_{i}, 0 \leq i \leq 2 p$, each containing $p$ edges $e_{i}^{0}, e_{i}^{1}, \ldots, e_{i}^{p-1}$. Then set

$$
c_{k}\left(e_{i}^{j}\right)=(i+(j+k)(2 p+1)) \bmod p(2 p+1) .
$$

Because of the choice of the modulo in the definition of the colouring $(p(2 p-1)$ for even $n$ and $p(2 p+1)$ for odd $n$ ), any colour is always in the same matching in all the $p$ strong colourings. This is the reason why the $p$ colourings are pairwise compatible (details of this part of the proof are left to the reader).

\subsection{Cartesian products of $(k, t)$-colourable graphs}

Theorem 6 Let $G$ be a $\left(k_{G}, t_{G}\right)$-colourable graph and let $H$ be a $\left(k_{H}, t_{H}\right)$-colourable graph, with $t_{G} \geq$ $\chi(H)$ and $t_{H} \geq \chi(G)$. Then $G \square H$ is $\left(k_{G}+k_{H}, \min \left(t_{G}, t_{H}\right)\right)$-colourable.

Proof: Let $t^{\prime}=\min \left(t_{G}, t_{H}\right)$. As $G$ is $\left(k_{G}, t_{G}\right)$-colourable, there exist $t_{G}$ compatible strong colourings $c_{i}, 1 \leq i \leq t_{G}$ of $G ; c_{i}: E(G) \rightarrow\left\{0,1, \ldots, k_{G}-1\right\}$. Similarly, as $H$ is $\left(k_{H}, t_{H}\right)$-colourable, there exist $t_{H}$ compatible strong colourings $d_{i}, 1 \leq i \leq t_{H}$ of $H$. Assume moreover that these colourings $d_{i}$ use different colours than the colourings of $G: d_{i}: E(H) \rightarrow\left\{k_{G}, k_{G}+1, \ldots, k_{G}+k_{H}-1\right\}$.

Let $v_{G}$ be a proper vertex colouring of $G$ using the $\chi(G)$ colours $0,1, \ldots \chi(G)-1$ and let $v_{H}$ be a proper vertex colouring of $H$ using the $\chi(H)$ colours $0,1, \ldots \chi(H)-1$.

We define the $t^{\prime}$ colourings $c_{i}^{\prime}, 0 \leq i \leq t^{\prime}-1$ of $G^{\prime}$ as follows: 
For any edge $a b$ of $G$, for any vertex $x$ of $H$, let $m(x)=\left(v_{H}(x)+i\right) \bmod t_{G}$ and set

$$
c_{i}^{\prime}((a, x)(b, x))=c_{m(x)}(a b),
$$

for any edge $x y$ of $H$, for any vertex $a$ of $G$, let $p(a)=\left(v_{G}(a)+i\right) \bmod t_{H}$ and set

$$
c_{i}^{\prime}((a, x)(a, y))=d_{p(a)}(x y) .
$$

Notice that each colouring $c_{i}^{\prime}$ uses at most $k_{G}+k_{H}$ colours.

Let us show that each $c_{i}^{\prime}$ is a strong colouring of $G^{\prime}$ :

First, consider two adjacent vertices $(a, x)$ and $(b, x)$ of $G^{\prime}$. Then $S_{c_{i}^{\prime}}((a, x)) \cap S_{c_{i}^{\prime}}((b, x))=\left(S_{c_{m(x)}}(a) \cup\right.$ $\left.S_{d_{p(a)}}(x)\right) \cap\left(S_{c_{m(x)}}(b) \cup S_{d_{p(b)}}(x)\right)$. Since $v_{G}$ is proper and $t_{G} \geq \chi(H)$, we have that $p(a) \neq p(b)$ and since $d_{p(a)}$ and $d_{p(b)}$ are compatible then we obtain $S_{d_{p(a)}}(x) \cap S_{d_{p(b)}}(x)=\emptyset$. Moreover, as $c_{m(x)}$ is strong, then we have $S_{c_{m(x)}}(a) \cap S_{c_{m(x)}}(b)=\left\{c_{m(x)}(a b)\right\}$. Consequently, $S_{c_{i}^{\prime}}((a, x)) \cap S_{c_{i}^{\prime}}((b, x))=$ $\left\{c_{m(x)}(a b)\right\}=\left\{c_{i}^{\prime}((a, x)(b, x))\right\}$.

Next, for two adjacent vertices $(a, x)$ and $(a, y)$ of $G^{\prime}$, a similar argument gives $S_{c_{i}^{\prime}}((a, x)) \cap S_{c_{i}^{\prime}}((a, y))=$ $\left\{d_{p(a)}(x y)\right\}=\left\{c_{i}^{\prime}((a, x)(a, y))\right\}$.

Now let us show that for any $i_{1}, i_{2}, 0 \leq i_{1}, i_{2} \leq t^{\prime}-1, i_{1} \neq i_{2}, c_{i_{1}}^{\prime}$ and $c_{i_{2}}^{\prime}$ are compatible: Let $(a, x)$ be a vertex of $G^{\prime}$. We have

$S_{c_{i_{1}}^{\prime}}((a, x))=S_{c_{m_{1}}}(a) \cup S_{d_{p_{1}}}(x)$, with $m_{1}=\left(v_{H}(x)+i_{1}\right) \bmod t_{G}$ and $p_{1}=\left(v_{G}(a)+i_{1}\right) \bmod t_{H}$, and

$S_{c_{i_{2}}^{\prime}}((a, x))=S_{c_{m_{2}}}(a) \cup S_{d_{p_{2}}}(x)$, with $m_{2}=\left(v_{H}(x)+i_{2}\right) \bmod t_{G}$ and $p_{2}=\left(v_{G}(a)+i_{2}\right) \bmod t_{H}$.

We have $m_{1} \neq m_{2}$ and $p_{1} \neq p_{2}$, therefore $S_{c_{m_{1}}}(a) \cap S_{c_{m_{2}}}(a)=\emptyset$ and $S_{d_{p_{1}}}(x) \cap S_{d_{p_{2}}}(x)=\emptyset$. Moreover, since for any $i$ and $k$, the colourings $c_{i}$ and $d_{k}$ do not use the same sets of colours, we conclude that $S_{c_{i_{1}}^{\prime}}((a, x)) \cap S_{c_{i_{2}}^{\prime}}((a, x))=\emptyset$.

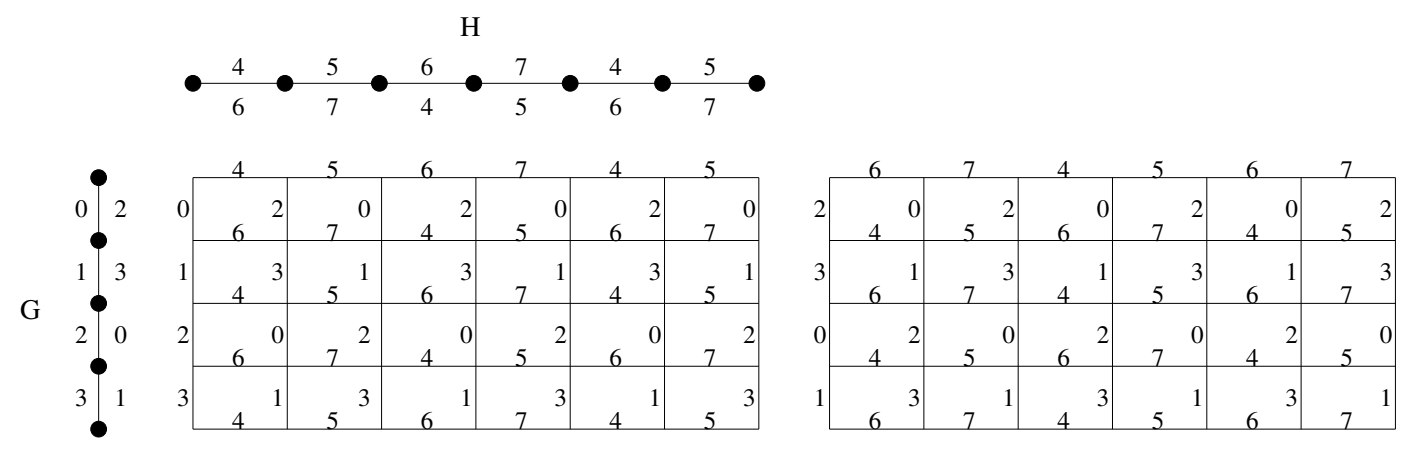

Fig. 2: Two compatible optimal strong colourings of $P_{5} \square P_{7}$ in 8 colours.

An example of two compatible strong colourings of $P_{5} \square P_{7}$ as defined in the above proof is given in Figure 2 (the two compatible strong colourings of $P_{5}$ and of $P_{7}$ appear on each graph but the vertex colourings are not given).

Corollary 1 Let $G$ be a $\left(k_{G}, t_{G}\right)$-colourable graph and let $H$ be a $\left(k_{H}, t_{H}\right)$-colourable graph. Then $G \square H$ is $\left(k_{G}\left\lceil\frac{\chi(H)}{t_{G}}\right\rceil+k_{H}\left\lceil\frac{\chi(G)}{t_{H}}\right\rceil, \min (\chi(G), \chi(H))\right)$-colourable. 
Proof: By definition, $G$ is $\left(k_{G}\left\lceil\frac{\chi(H)}{t_{G}}\right\rceil, \chi(H)\right)$-colourable and $H$ is $\left(k_{H}\left\lceil\frac{\chi(G)}{t_{H}}\right\rceil, \chi(G)\right)$-colourable. Theorem 6 then gives the result.

Corollary 2 Let $G$ be a $(k, 2)$-colourable graph. Then $G \square K_{2}$ is $(k+\chi(G), 2)$-colourable.

\subsection{Products of paths, cycles and cliques}

The $d$-dimensional grid $M_{l_{1}, l_{2}, \ldots, l_{d}}$ is the Cartesian product of $d$ paths: $M_{l_{1}, l_{2}, \ldots, l_{d}}=P_{l_{1}} \square P_{l_{2}} \square \ldots \square P_{l_{d}}$. When all lengths are equal: $l_{1}=l_{2}=\ldots=l_{d}=n$, we denote the grid by $M_{n}^{d}$.

The $d$-dimensional toroidal grid $T M_{l_{1}, \ldots, l_{d}}$ is the Cartesian product of $d$ cycles: $T M_{l_{1}, \ldots, l_{d}}=C_{l_{1}} \square \ldots \square C_{l_{d}}$. When all cycle lengths are equal: $l_{1}=l_{2}=\ldots=l_{d}=n$, we denote the toroidal grid by $T M_{n}^{d}$.

The $d$-dimensional generalized hypercube (also known as Hamming graph) $K_{n}^{d}$ is the Cartesian product of $K_{n}$ by itself $d$ times: $K_{n}^{d}=K_{n} \square K_{n} \square \ldots \square K_{n}$. The hypercube $H_{d}$ is the graph $K_{2}^{d}$.

By Proposition 1 and Proposition 2, $P_{n}$ and $C_{4 n}$ are $(4,2)$-colourable and $C_{2 n}$ is $(5,2)$-colourable for $n \neq 3$. By Proposition 3 , $K_{2 p}$ is $(2 p(2 p-1), 2 p)$-colourable and $K_{2 p+1}$ is $(3 p(2 p+1), 2 p+1)$-colourable. Therefore, Theorem 6 and Corollary 2 give the following results:

Corollary 3 For any $d \geq 2$,

- the d-dimensional hypercube $H_{d}$ is $(2 d, 2)$-colourable,

- the d-dimensional grid $M_{l_{1}, l_{2}, \ldots, l_{d}}$ and toroidal grid $T M_{4 n}^{d}$ are $(4 d, 2)$-colourable,

- the d-dimensional toroidal grid $T M_{2 l_{1}, 2 l_{2}, \ldots, 2 l_{d}}$ is $(5 d, 2)$-colourable for $l_{i} \neq 3$,

- the d-dimensional generalized hypercube $K_{2 p}^{d}$ is $(2 d p(2 p-1), 2 p)$-colourable,

- the d-dimensional generalized hypercube $K_{2 p+1}^{d}$ is $(3 d p(2 p+1), 2 p+1)$-colourable.

With Theorem 11, we obtain exact or approximate values for the strong chromatic index of some Cartesian products:

Corollary 4 For any $d \geq 2$,

- $\chi_{s}^{\prime}\left(H_{d}\right)=2 d$,

- $\chi_{s}^{\prime}\left(M_{l_{1}, l_{2}, \ldots, l_{d}}\right)=\chi_{s}^{\prime}\left(T M_{4 n}^{d}\right)=4 d$, for $l_{i} \geq 3$,

- $4 d \leq \chi_{s}^{\prime}\left(T M_{2 l_{1}, 2 l_{2}, \ldots, 2 l_{d}}\right) \leq 5 d$, for $l_{i} \neq 3$,

- $2 p(d-1)(2 p-1) \leq \chi_{s}^{\prime}\left(K_{2 p}^{d}\right) \leq 2 d p(2 p-1)$,

- $2 p(d-1)(2 p+1) \leq \chi_{s}^{\prime}\left(K_{2 p+1}^{d}\right) \leq 3 d p(2 p+1)$.

Note that the exact value of the strong chromatic index of hypercubes was known before [2]. 


\section{References}

[1] L. D. Andersen. The strong chromatic index of a cubic graph is at most 10. Discrete Math., 108(13):231-252, 1992. Topological, algebraical and combinatorial structures. Frolík's memorial volume.

[2] R. J. Faudree, R. H. Schelp, A. Gyárfás, and Zs. Tuza. The strong chromatic index of graphs. Ars Combin., 29(B):205-211, 1990. Twelfth British Combinatorial Conference (Norwich, 1989).

[3] P. Gvozdjak, P. Horák, M. Meszka, and Z. Skupień. Strong chromatic index for multigraphs. Util. Math., 57:21-32, 2000.

[4] J. Janssen and L. Narayanan. Approximation algorithms for channel assignment with constraints. Theoret. Comput. Sci., 262(1-2):649-667, 2001.

[5] M. Mahdian. The strong chromatic index of $C_{4}$-free graphs. In Proceedings of the Ninth International Conference "Random Structures and Algorithms" (Poznan, 1999), volume 17(3-4), pages 357-375, 2000.

[6] M. Mahdian. On the computational complexity of strong edge coloring. Discrete Appl. Math., 118(3):239-248, 2002.

[7] M. Molloy and B. Reed. A bound on the strong chromatic index of a graph. J. Combin. Theory Ser. $B, 69(2): 103-109,1997$.

[8] J. J. Quinn and A. T. Benjamin. Strong chromatic index of subset graphs. J. Graph Theory, 24(3):267-273, 1997.

[9] J. J. Quinn and E. L. Sundberg. Strong chromatic index in subset graphs. Ars Combin., 49:155-159, 1998.

[10] H. Tamura, K. Watanabe, M. Sengoku, and S. Shinoda. Graph theoretical considerations of a channel assignment problem on multihop wireless networks. In Proceedings of ITC-CSCC conference (Phuket, Thailand), 2002. 